\title{
User Experience in Cars
}

\author{
Manfred Tscheligi ${ }^{1}$, Albrecht Schmidt ${ }^{2}$, David Wilfinger ${ }^{1}$, \\ Alexander Meschtscherjakov ${ }^{1}$, and Andrew L. Kun ${ }^{3}$ \\ ${ }^{1}$ ICT\&S Center, University of Salzburg, 5020 Salzburg, Austria \\ \{manfred.tscheligi, david.wilfinger, \\ alexander.meschtscherjakov\} asbg.ac.at \\ ${ }^{2}$ University of Stuttgart, 70569 Stuttgart, Germany \\ albrecht.schmidt@acm.org \\ ${ }^{3}$ University of New Hampshire, Electrical and Computer Engineering Department, \\ Kingsbury Hall, Durham, NH 03824, USA \\ andrew. kun@unh.edu
}

\begin{abstract}
This workshop will address two emerging fields within the HCI community: user experience (UX) and the automotive context. It will bring HCI experts together to discuss UX factors for the specific characteristics of car interiors and automotive user interfaces. It targets the development of a better view of UX within the whole car (driver, front seat, backseat area) beyond traditional marketing instruments known within the automotive industry.
\end{abstract}

\section{Topic, Objectives and Outcomes}

$\mathrm{HCI}$ is always dependent on the context where the interaction happens and therefore contingent on a multitude of factors. Especially the automotive context has its own and very specific characteristics. Car cockpits have become more complex due to a variety of assistance systems and infotainment capabilities. In addition cars are often a very emotional topic for humans. This workshop will explore the inclusion of user experience (UX) aspects in researching and developing automotive user interfaces.

With cars becoming interactive systems that offer besides transportation all kinds of information access, assistance, as well as entertainment, UX research has become a major challenge in this context. Alternative interaction means for example will be offered for the driver as well as for front and rear seat passengers (e.g. supporting transparency related to the driving situations or supporting co-pilot activities). The introduction of such interactive systems will require a deep understanding of UX in the car (i.e. car as a very special context) in order to be successful. For that purpose a common understanding of user experience and its effects on car technology (and all different subparts) usage is needed, as well as an in depth discussion on the applicability of UX methods that were established in other application contexts. This workshop therefore addresses the following issues:

- Understanding of UX in the automotive context in a holistic way

- Looking into different sub-domains: the driver, the front seat, the backseat area, interconnection to the outside world

- Researching the relevance of traditional UX factors in the automotive context and identification of new UX factors especially relevant for the car 
- Contextual analysis and evaluation methods for UX in the automotive context

- Discussion of car laboratory setups in comparison to in-situ methods for car UX research

- Automotive user interface frameworks and toolkits

- New concepts for in-car user interfaces increasing UX and supporting safety

- Experience design in the car

- Looking into the future: the ubiquitous car in a mobile society

The intended outcomes of this workshop include the creation of a common understanding and perspective of the challenges related to UX and automotive interfaces. For that purpose the workshop will discuss perspectives on how to address these challenges in future research and development. After the workshop accepted papers will be made available on the workshop website. The publication of a revised version of papers as a special issue of a journal (such as Personal and Ubiquitous Computing or Interacting with computers) will be considered and an Edited Book is envisaged (see more details on http://workshops.icts.sbg.ac.at/interact2011).

\section{Workshop Organization}

The workshop is intended for: HCI researcher in general, who are interested specifically in the automotive context; UX experts, who are willing to adapt UX models and extend UX factors for the specific characteristics of interaction in the car; automotive user interface designers and engineers both from a scientific, as well as from an industrial perspective. Workshop candidates are requested to send a position paper about their research and link to the workshop theme to the organizers (email: interact2011@hciunit.org).

This is a one-day workshop with breakout sessions, alternated with a moderated group discussion. The workshop will start with an introduction to the workshop topic, followed by introductory presentations. After a break the organizers present the common themes of the submitted papers, grouping them into different sessions. The different groups will then discuss their topics during a first breakout session. After the lunch break a short alignment of pre-lunch discussions will happen in the whole group. This continues into a second round of breakout sessions to further discuss grouped topics. After a coffee break the results of the breakout sessions will be discussed in the whole group. The conclusions of the workshop will be worked out and follow up activities will be specified.

Organizers' backgrounds: Manfred Tscheligi is professor for HCI \& Usability at the University of Salzburg. He is currently acting as Conference Chair for the $3^{\text {rd }}$ Conference AutomotiveUI 2011. Albrecht Schmidt is a professor for Human-Systems Interaction and Cognitive Systems at the University of Stuttgart and was Conference Co-Chair of AutomotiveUI 2010 and 2009. Andrew Kun is Associate Professor of Electrical and Computer Engineering at the University of New Hampshire and was Program Co-Chair of AutomotiveUI 2010. Alexander Meschtscherjakov and David Wilfinger are Research Fellows at the HCI Unit of the ICT\&S Center (University of Salzburg) working in the area of automotive user interfaces. 